\title{
La Voce dei Pazienti \\ Certe luci non puoi spegnerle
}

Questa toccante testimonianza di Sabrina ci aiuta a capire che la vita è una costante sfida alla nostra capacità di resistere e di reagire alle avversità. A momenti felici si alternano momenti di buio e la luce in fondo al tunnel sembra non arrivare mai. Ma noi siamo fatti di una materia complessa, in cui il dolore e la capacità di reagire possono e devono convivere per creare un delicato equilibrio. Alla notte segue sempre il giorno e quello che arriva potrebbe, finalmente, essere un giorno di speranza.

\section{"Certe luci non puoi spegnerle."}

Questa parole, scritte da Ligabue, accompagnano la mia vita da molti anni.

La luce è vita, speranza, rinascita. È l’alternarsi del giorno alla notte, i due volti della stessa medaglia. È il coraggio di rialzarsi dopo essersi seduti ed è la spinta a riprendere a camminare dopo essersi rialzati.

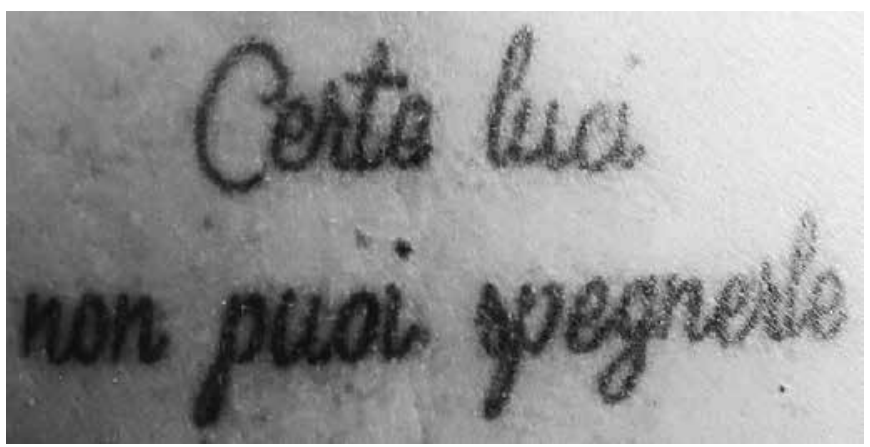

La mia luce è anche il mio Angelo custode: si chiama Giorgia, da 17 anni brilla in cielo e veglia su di me da lassù. Ha il volto e la dolcezza di una bambina, mia figlia, che all'età di sei mesi dalla nascita manifestò i sintomi di una rarissima malattia genetica metabolica mitocondriale alla quale sopravvisse, andando oltre i sei mesi di vita previsti dai medici, per ben cinque anni. Le successive analisi genetiche stabilirono che tanto io quanto mio marito siamo portatori sani di questa malattia e pertanto da qui scaturì la decisione di adottare un bambino.

Dopo il buio più cupo di una esistenza-non esistenza lunga cinque anni, tornò finalmente la luce nella vita di Sabrina e Roberto: questa volta con il volto di un bambino dal nome di Simone.

Simone è oggi un ragazzone di 16 anni, volenteroso, affettuoso, sbadato, spensierato, testardo, con gli ormoni impazziti e con tutti i pregi e i difetti di un adolescente.
Ma il ciclo della vita corrisponde al ciclo del mondo su cui le nostre storie quotidiane scorrono: la notte insegue sempre il giorno.

E la notte giungeva puntuale in seguito a un banalissimo atto di bullismo subito da mio figlio: una semplice ecografia all'addome escludeva qualsiasi danno causato da un pugno ricevuto nel ventre ma evidenziava senza ombra di dubbio una malattia genetica, latente nei reni di Simone e in modo subdolo finora asintomatica e da me misconosciuta: il rene policistico autosomico dominante.

La notte rincorre il giorno così come la storia, nel suo ripetersi, insegue se stessa.

Esami, consulti, visite mediche specialistiche contribuivano a prendere sempre maggior coscienza con questa malattia e contemporaneamente allungavano la durata della notte. La sempre maggior consapevolezza di quanto si stava nuovamente affrontando, l'impotenza davanti all'ennesima malattia incurabile, la frustrazione causata dalla scoperta di un nuovo medicinale efficace e utilizzato in gran parte del mondo ma assurdamente non autorizzato dall'Agenzia del Farmaco Italiana. Le notti tormentate dai pensieri che si accavallavano stratificandosi come nubi plumbee foriere di un violento temporale. L'illusione che, addormentandosi, ci si possa poi svegliare da un brutto sogno sostituitosi alla realtà per qualche assurdo motivo.

Perché? Perché nuovamente mio figlio? Perché nuovamente noi? Ho già pagato alla vita un pedaggio eterno: chi è che mi vuole tanto male?

"Ciao mio dolce angelo, ti devo chiedere un grande favore e mi rivolgo a te che, durante il tuo lustro trascorso su questo pianeta, hai patito le peggiori pene che si possano solo immaginare. Ora, come già sai, hai un fratellone che, sebbene in modo meno grave rispetto a te, ha incontrato sulla sua strada una malattia abbastanza rara e pertanto, anche lui, sarà destinato in futuro a soffrire parecchio. Potresti da lassù far qualcosa? Perché io da quaggiù ora sono proprio in difficoltà! Grazie!"

Questa volta, e mai come questa volta, l'aurora tardava ad arrivare. 
Alla ricerca di un barlume di luce che potesse finalmente portare un po' di calore nell'anima, nel mese di marzo 2016 scrissi, priva di illusioni, a Massimo Gramellini, allora vicedirettore del quotidiano La Stampa e ora vicedirettore del Corriere della Sera. Scelsi lui perché sono sempre stata attratta dai suoi articoli e soprattutto perché sovente le sue parole giungono dritte al cuore. E lo feci più per il bisogno di sfogare il mio stato di impotenza che per la speranza di vedere una luce in fondo al tunnel: quando si precipita nel baratro, ogni appiglio può essere utile a frenare la caduta. Consapevole che Gramellini possa ricevere quotidianamente centinaia di lettere, venni colta di sorpresa quando, nel mese di settembre, mi giunse la sua risposta. Si dimostrò interessato e scrisse che avrebbe trasmesso il mio caso a un suo amico e collega: Giacomo Galeazzi. Quest'ultimo, giornalista e autore di numerosi libri, dopo aver appreso da me e dai suoi fitti contatti tutte le informazioni a lui necessarie, pubblica, domenica 12 marzo 2017 sul quotidiano La Stampa, una minuziosa inchiesta in merito alla malattia e sulla sua cura esistente ma negata ai pazienti italiani. L'eco dell'articolo rimbalza immediatamente sui telegiornali nazionali e si diffonde presso altre testate giornalistiche: uno tsunami che di ora in ora avanza acquistando potenza e dimensioni.
Il giorno successivo giunge come naturale conseguenza la notizia attesa oramai da troppo tempo: I'AIFA ha autorizzato I'uso del farmaco per 1800 ammalati di rene policistico.

L'alba. Ciò che più ho desiderato. I miei occhi, lucidi per la gioia e colmi di speranza, vedono finalmente la luce di un nuovo splendido giorno.

No, certe luci non si possono proprio spegnere.

Grazie Massimo Gramellini.

Grazie Giacomo Galeazzi.

Grazie Giorgia.

Sabrina

Grazie, Sabrina.

Luisa Sternfeld Pavia

Presidente AIRP

Published online: May 25, 2017 